\title{
Riddles in perovskite research
}

Metal halide perovskites are not only excellent materials for photovoltaic and light-emitting applications they are also an intriguing riddle for researchers trying to understand the relationship between structure and optoelectronic properties in these semiconductors. A very broad range of experimental techniques, as well as computer simulations, have been used to extract key charge transport parameters, such as diffusion lengths, lifetimes, recombination rates and effective masses of the charge carriers, sometimes leading to behaviour unexpected for solution-made, inherently soft materials. We asked four teams of researchers to share their views on the current understanding of the photophysics of perovskites. They discuss some of the properties and effects - such as structural fluctuations, lattice softness, ferroelectric-like large polarons, Rashba effects and defect photochemistry - that may make these semiconductors different from other materials used in optoelectronics. Moreover, they suggest further investigations to clarify the relative impact of these properties on the performance of solar cells, and propose ways to exploit some of these effects in other devices based on metal halide perovskites.

\section{Entropy in halide perovskites}

\author{
Claudine Katan, Aditya D. Mohite and Jacky Even discuss the possible impact of various entropy contributions \\ (stochastic structural fluctuations, anharmonicity and lattice softness) on the optoelectronic properties of halide \\ perovskite materials and devices.
}

$\mathrm{H}$ alide perovskites have undeniably remarkable characteristics, which are interesting for next-generation technologies. Yet results that suggest moderate diffusion lengths and mobilities for their charge carriers are hard to reconcile with other experimental observations pointing towards long carrier lifetimes, low effective masses of carriers or short Urbach tails in the absorption spectrum. Moreover, exciton screening at room temperature, hot carrier effects and the relaxation of carriers toward the band edges are other important microscopic processes that remain poorly understood. Providing a clear picture of the behaviour of these materials has proved challenging, partly because all the abovementioned optoelectronic properties are affected by the unusually strong structural fluctuations undergone by halide perovskites.

In classic semiconductors, structural fluctuations are usually represented by a thermal bath of phonons, an ensemble of quasiparticles each representing a quantized vibration at a specific frequency. In these systems, atomic displacements away from equilibrium are small, and thus the vibrational density of states is described within the harmonic approximation, with the energy potential function showing a single minimum around the equilibrium position (Fig. 1a, blue line). Small departures from this ideal behaviour, known as weak anharmonicity (Fig. 1a, red line), result in phonon-phonon collisions that lead to temperature-dependent phonon linewidths and non-zero thermal conductivity.

In contrast, charge carriers moving in hybrid perovskite compounds find a highly perturbed electrostatic landscape, owing to stochastic (random) structural fluctuations of the organic and inorganic components and, more broadly, to all the sources of lattice softness ${ }^{1}$. These phenomena and their impact on the optoelectronic properties of halide perovskites have received little scrutiny. Stochastic structural fluctuations have two origins. First, the energy potential of the halide-perovskite lattice shows a double-well, strongly anharmonic shape (Fig. 1a, green line, and Fig. 1b), leading to disordered motion of the halides perpendicular to the metal-metal axis ${ }^{2,3}$; second, the charge anisotropy of the organic cations (for instance, methylammonium, $\mathrm{MA}^{+}$, or formamidinium, $\mathrm{FA}^{+}$) leads them to move in a disordered tumbling fashion within the cuboctahedral lattice cavities (Fig. 1c). Frozen at low temperature, these organic dipoles keep disordered orientations, a glassy behaviour that has been predicted theoretically ${ }^{1}$ and observed through dielectric and thermodynamic experiments. ${ }^{4}$ These unconventional features are reminiscent of atomic disorder observed in two other well-known classes of materials: plastic crystals ${ }^{5}$ and oxide perovskites ${ }^{6}$.

At a basic level, the direct impact of stochastic cation reorientations on the carrier transport processes can be gauged in the framework of the 'dipolar 
a

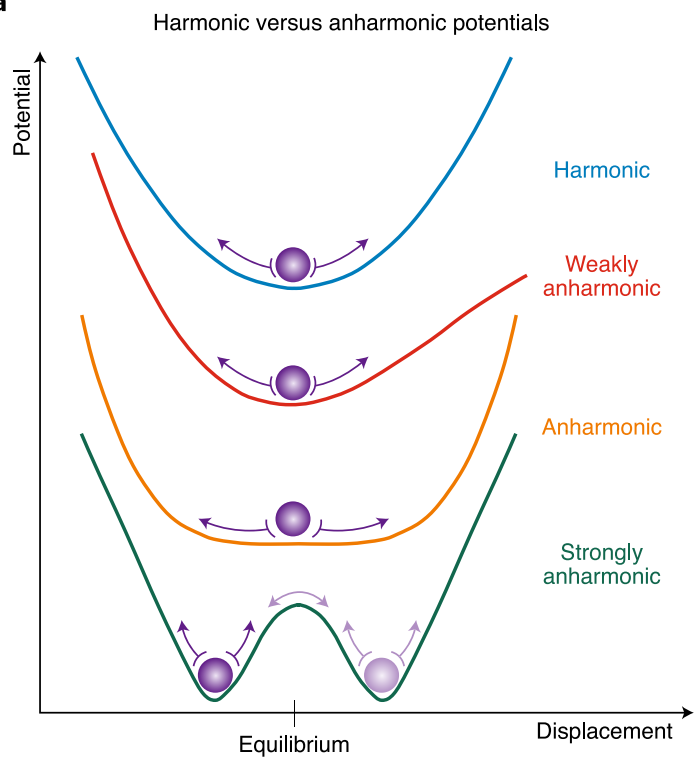

b

Anharmonic motion of halide

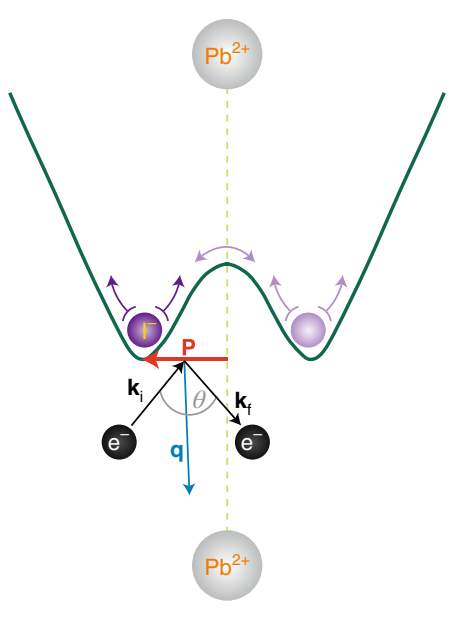

c

Motion of organic cation
Transverse acoustic phonon

e
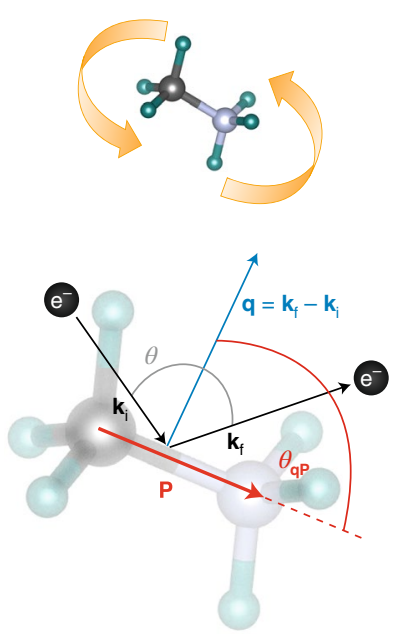

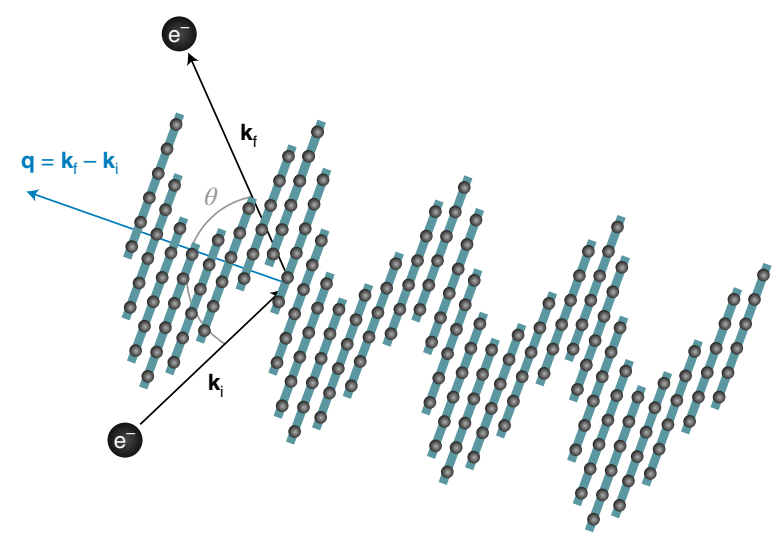

Longitudinal acoustic phonon

Fig. 1 | Sources of dynamic disorder in halide perovskites. a, Various shapes of energy potentials, from harmonic to strongly anharmonic. The violet sphere represents an atom moving in the potential around its equilibrium position. b-e, Scattering events that lead a charge (electron or hole, black sphere) to change its wavevector from $\mathbf{k}_{\mathrm{i}}$ to $\mathbf{k}_{\mathrm{f}}$ owing to its interaction with (b) the anharmonic motion of a halide atom (in the example shown, the violet sphere represents an iodine atom; the dashed line is a guide to the eye to locate the equilibrium position on the axis connecting the two lead atoms); (c) the stochastic tumbling motion of the organic cation (an $\mathrm{MA}^{+}$cation is shown in the example, with carbon, nitrogen and hydrogen atoms shown as grey, light-blue and green spheres, respectively); (d) transverse and (e) longitudinal acoustic phonon modes, respectively represented as shear and compression waves moving the perovskite lattice planes (green-shaded regions with grey spheres representing atom positions). $\mathbf{P}$ is the electric dipole, $\mathbf{q}=\mathbf{k}_{\mathrm{f}}-\mathbf{k}_{\mathrm{i}}$ the elastic change in wavevector, $\theta_{\mathrm{qP}}$ the angle between vectors $\mathbf{P}$ and $\mathbf{q}, \theta$ the angle between $\mathbf{k}_{\mathrm{f}}$ and $\mathbf{k}_{\mathrm{i}}$.

scattering' mechanism. This specific microscopic mechanism, rarely considered for classical semiconductors, provides a crude description of carrier scattering by uncorrelated random dipolar fluctuations (Fig. 1b,c) ${ }^{7}$. Its direct influence on carrier transport can be evaluated by computing the dipolar scattering relaxation time $\left(\tau_{\mathrm{d}}\right)$ in the effective mass $(m)$ approximation, $\tau_{\mathrm{d}}\left(E / k_{\mathrm{B}} T\right)=\frac{2 \pi \varepsilon \hbar^{2} \sqrt{2 k_{\mathrm{B}} T}}{P^{2} e^{2} N_{\mathrm{p}} \sqrt{m}\left(\cos \left(\theta_{\mathrm{q}}\right)\right)^{2}} \frac{\sqrt{E / k_{\mathrm{B}} T}}{g\left(E / k_{\mathrm{B}} T\right)}$, where $E$ is the carrier energy, $g$ a function describing the screening of the electrostatic interaction, $\theta_{\mathrm{qP}}$ the angle between the fluctuating electric dipole $\mathbf{P}$ and vector $\mathbf{q}$ defining the elastic change in wavevector (Fig. 1c), $P$ the magnitude of the dipole, $N_{\mathrm{P}}$ the dipole concentration, $\hbar$ the reduced Planck constant, $k_{\mathrm{B}}$ the Boltzmann constant, $T$ the temperature, $\varepsilon$ the dielectric constant and $e$ the electric charge ${ }^{8}$.

The carrier mobility is then obtained by numerically integrating over the thermal distribution of carriers. For low carrier concentrations (such as $10^{14} \mathrm{~cm}^{-3}$ in the weak screening regime), a computed maximum mobility as high as about $900 \mathrm{~cm}^{2} \mathrm{~V}^{-1} \mathrm{~s}^{-1}$ for $\mathrm{MAPbI}_{3}$ (considering $m=0.21, \varepsilon=6$ and
$P=2.85$ debye) can be reached by taking into account disordered reorientations of $\mathrm{MA}^{+}$. This value is considerably higher than the mobilities experimentally measured in this material, suggesting that the dipolar scattering of charge carriers induced by the organic cations is unlikely to be the limiting factor in MA-based perovskites - and will similarly have no role in $\mathrm{FAPbI}_{3}$ or $\mathrm{CsPbI}_{3}$, whose cations have small or vanishing electric dipole.

The most remarkable differences between halide perovskites and classic semiconductors stem from the phonon 
bath itself, mostly related to the fluctuations of the halide perovskite lattice. This exhibits a very low stiffness ${ }^{9}$ and large anharmonicity ${ }^{2}$, and more generally the phonon vibrational density of states lies at a lower energy, thus promoting electronphonon collision processes (Fig. 1b and Fig.1d,e). In such cases, perturbative approaches break down and more complex theoretical strategies, such as the stochastic self-consistent harmonic approximation, must be considered. For example, in $\mathrm{CsSnI}_{3}$ perovskite, a proper treatment of phonon-phonon interactions using such a method revealed huge modifications of the vibrational density of states and, in turn, sizable renormalization of the electronic bandgap ${ }^{2}$. Insights into these effects on monoelectronic states close to the bandgap and more generally on the optoelectronic properties of halide perovskites deserve further theoretical and experimental investigation ${ }^{10}$. Alternatively, a more basic understanding of the impact on carrier mobility of large ionic displacements can be gained from the dipolar scattering model by considering the dipole induced by the motion of the halide atoms (Fig. 1b). For instance, an iodine displacement of $0.4 \AA$ (ref. ${ }^{3}$ ) leads to a considerable decrease in the maximum carrier mobility allowed (about $160 \mathrm{~cm}^{2} \mathrm{~V}^{-1} \mathrm{~s}^{-1}$ ); this suggests that dipolar fluctuations due to the anharmonicity of the halide motion have a stronger impact on the behaviour of charges than the fluctuations due to the organic cations. Yet it is important to mention that the latter may have an additional indirect impact: in hybrid perovskites, molecular rotations combine with the lattice strain to renormalize the elastic constants, as evidenced by optical spectroscopy ${ }^{9}$, and may thus affect the halide motion.

The understanding of the effects of lattice softness can also benefit from earlier work on oxide perovskites, where order-disorder effects coexist with displacive dynamics ${ }^{6}$, and low-frequency polar optical modes, corresponding to the above-mentioned halide vibrations (Fig. 1b), strongly interact through nonlinear local coupling with acoustic modes (Fig.1d,e). Organic and inorganic halide perovskites undergo similar coupling mechanisms ${ }^{1,9}$. Additional investigations are needed to monitor the importance of the acoustic phonon anharmonicity and of the extreme lattice softness on carrier scattering processes. The presence of unusual phonon bottlenecks that slow down the cooling of charge carriers may be another consequence of sizable anharmonicity and low-lying vibrational density of states of the perovskite lattice ${ }^{11}$. Its impact on hot carrier effects and on carrier mobilities requires further elucidation.

The same holds for the strong coupling regime associated to non-polar interactions between local distortions and charge carriers, which leads to the formation of small polarons $^{12}$. This phenomenon may lead to the formation of two types of charge carriers that have different lifetimes, effective masses and other characteristics. Organic cations seem to favour formation of small polarons. They may also contribute to the ultralow heat conductivities of hybrid perovskites ${ }^{11}$.

It is the study of heat conduction, as well as of other macroscopic material properties depending on low-energy relaxation processes and the vibrational density of states (such as specific heat, mechanical stiffness or dielectric susceptibility), that can improve the microscopic understanding of halide perovskites and provide guidance to optimize their use in optoelectronic devices. Among the recent experimental attempts along this path are detailed studies on the light-induced emergence and activation of molecular rotation, electron-phonon interactions and polaron formation ${ }^{13-15}$.
The building up of such interactions in the context of operating devices will provide further insight on metastability and nonequilibrium phenomena in perovskites.

\section{Claudine Katan ${ }^{1 \star}$, Aditya D. Mohite ${ }^{2,3}$ and Jacky Even ${ }^{4 *}$ \\ ${ }^{1}$ Univ Rennes, ENSCR, INSA Rennes, CNRS, ISCR (Institut des Sciences Chimiques de Rennes) - UMR, 6226 Rennes, France. ${ }^{2}$ Department of Chemical and Biomolecular Engineering, Rice University, Houston, TX, USA. ${ }^{3}$ Division of Materials Physics and Application, Los Alamos National Laboratory (LANL), Los Alamos, NM, USA. ${ }^{4}$ Univ Rennes, INSA Rennes, CNRS Institut FOTON - UMR, 6082 Rennes, France. *e-mail: claudine.katan@univ-rennes1.fr; jacky.even@insa-rennes.fr}

Published online: 23 April 2018

https://doi.org/10.1038/s41563-018-0070-0

References

1. Even, J., Carignano, M. \& Katan, C. Nanoscale 8, 6222-6236 (2016).

2. Patrick, C. E., Jacobsen, K. W. \& Thygesen, K. S. Phys. Rev. B 92, 201205 (2015).

3. Marronnier, A. et al. J. Phys. Chem. Lett. 8, 2659-2665 (2017).

4. Fabini, D. H. et al. J. Phys. Chem. Lett. 7, 376-381 (2016).

5. Lynden-Bell, R. M. \& Michel, K. H. Rev. Mod. Phys. 66 , 721-762 (1994)

6. Bussmann-Holder, A., Büttner, H. \& Bishop, A. R. Phys. Rev. Lett. 99, 167603 (2007).

7. Boardman, A. D. Proc. Phys. Soc. 85, 141-148 (1965).

8. Senanayak, S. P. et al. Sci. Adv. 3, el601935 (2017).

9. Létoublon, A. et al. J. Phys. Chem. Lett. 7, 3776-3784 (2016)

10. Giustino, F. Rev. Mod. Phys. 89, 015003 (2017).

11. Yang, J. et al. Nat. Commun. 8, 14120 (2017).

12. Neukirch, A. J. et al. Nano Lett. 16, 3809-3816 (2016).

13. Wu, X. et al. Sci. Adv. 3, el602388 (2017).

14. Ghosh, T., Aharon, S., Etgar, L. \& Ruhman, S. J. Am. Chem. Soc. 139, 18262-18270 (2017).

15. Taylor, V. C. A. et al. J. Phys. Chem. Lett. 9, 895-901 (2018).

\section{Acknowledgements}

In memory of G. C. Papavassiliou. The work at Los Alamos National Laboratory (LANL) was supported by the LDRD programme (A.D.M.). Part of the work was conducted at the Center for Integrated Nanotechnologies (CINT), a US Department of Energy Office of Science user facility. The work in France was supported by Agence Nationale pour la Recherche (TRANSHYPERO project). ADM acknowledges the DOE_EERE-0001647-1544 programme for funding for this work.

\title{
Ferroelectric large polarons
}

\author{
Kiyoshi Miyata and X.-Y. Zhu analyse the ferroelectric-like dielectric response of lead halide perovskites in the \\ terahertz region and discuss the potential role of polar nanodomains in accounting for the defect tolerance and low \\ recombination rates of these materials.
}

ead halide perovskites (LHPs) are remarkable for their electronic properties such as long charge-carrier lifetimes and diffusion lengths. Among the proposals to explain these exceptional carrier properties, the large polaron mechanism ${ }^{1,2}$ assumes that the screened Coulomb potential reduces carrier scattering from charged defects, other charge carriers and the remaining phonon bath. A conventional large polaron model ${ }^{2,3}$ assumes that the polarization $P$ of the ionic lattice - the dipole moment per unit volume resulting from atomic displacements from the equilibrium position - shows a linear response to an electric field $E_{\mathrm{f}}$ applied externally or generated by an extra charge introduced into the material (Fig. 1a, left). However, atomic motions, or phonons, in LHPs are known to be anharmonic and dynamically disordered ${ }^{4}$ and should show peculiar characteristics that lead to more complex relationships between $P$ and $E_{\mathrm{f}}$ (Fig. 1b, left, discussed below), altering this conventional picture. 

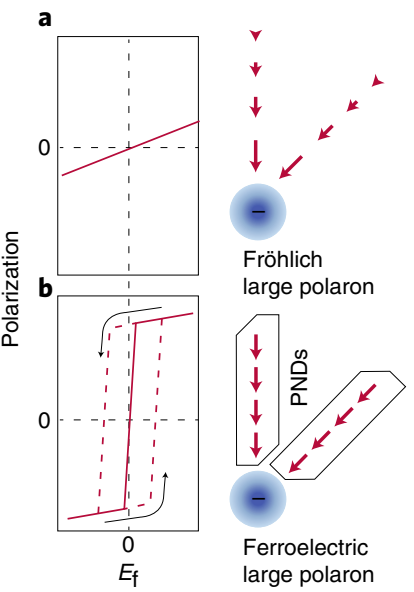

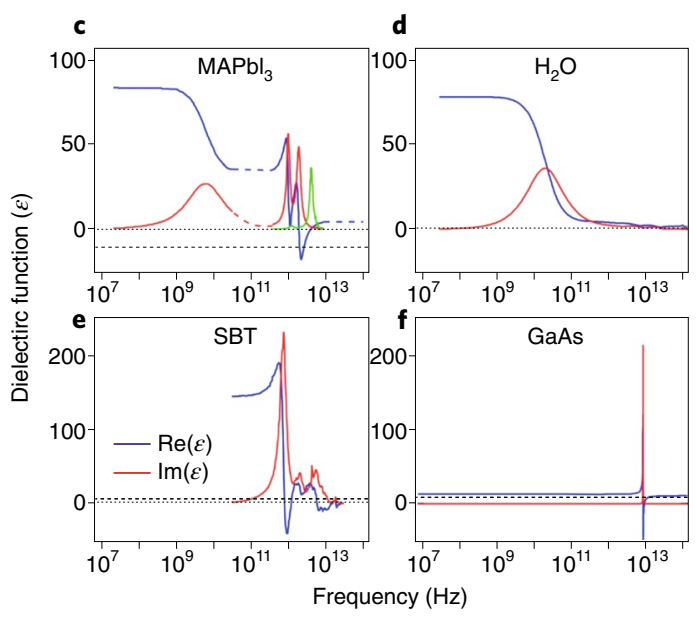

Fig. 1 | Ferroelectric-like behaviour of lead halide perovskites and implications for the large polaron model. a,b, Polarization response to an electric field $E_{\mathrm{f}}$ in a conventional crystal ( $\mathbf{a}$, left) and a ferroelectric-like material ( $\mathbf{b}$, left). The red dashed lines in $\mathbf{b}$ represent the response when the potential barrier between two minima of the potential energy surface is above thermal energy $k T$. Panels on the right show a Fröhlich large polaron (a, right) in which the dipole moment in a unit cell (red arrow) in the presence of an extra charge (blue sphere) decreases with the distance from the charge, and a ferroelectric large polaron (b, right), in which dipole moments in a polar nanodomain (PND) are uniformly affected by the charge. $\mathbf{c - f}$, Real ( $\varepsilon_{1}$, blue) and imaginary ( $\varepsilon_{2}$, red) dielectric functions of four materials. c, Hybrid perovskites $\mathrm{MAPbl}_{3}$ in tetragonal phase. The green curve (magnified by a factor of 20 ) is the LO phonon-absorption spectrum given by $\operatorname{Im}[-1 / \varepsilon(\nu)]$. d, Liquid water. e, Ferroelectric $\mathrm{SrBi}_{2} \mathrm{Ta}_{2} \mathrm{O}_{9}(\mathrm{SBT})$. f, Conventional semiconductor GaAs.Credit: adapted from ref. ${ }^{3}$, Royal Society of Chemistry and ref. ${ }^{5}$, Wiley (c); ref. ${ }^{14}$, Univ. of Missouri-Kansas City (d); ref. ${ }^{15}$, AIP (e); ref. ${ }^{16}$, Academic (f).

To understand how such phonon behaviour affects the formation and properties of large polarons, we examine the dielectric function of LHPs, $\varepsilon(\nu)=\varepsilon_{1}(\nu)+\mathrm{i} \varepsilon_{2}(\nu)$, where $\varepsilon_{1}$ describes polarization and $\varepsilon_{2}$ absorption, $\nu$ is frequency. Polarization is related to the dielectric function by $\operatorname{Re}(P)=\varepsilon_{0}\left(\varepsilon_{1}-1\right) E_{\mathrm{f}}$, where $\varepsilon_{0}$ is the vacuum permittivity. At frequencies above about $10 \mathrm{THz}, \varepsilon(\nu)$ is mainly determined by valence-electron densities, whereas at frequencies below $10 \mathrm{THz}$ it reflects how phonons respond to an external electric field. We show $\varepsilon(\nu)$ of methylammonium lead iodide $\left(\mathrm{MAPbI}_{3}\right)$ in Fig. 1c. In the frequency range of $10^{7} \mathrm{~Hz}$ to $10^{14} \mathrm{~Hz}, \varepsilon_{1}$ changes in two steps: the first at about $6 \mathrm{GHz}\left(\nu_{\text {rot }}\right)$ when $\varepsilon_{1}$ doubles because of reorientational relaxation of dipolar $\mathrm{MA}^{+}$ cations $^{3,5}$, and the second at about $1 \mathrm{THz}$ when $\varepsilon_{1}$ increases by an order of magnitude following the excitation of transverse optical (TO) phonon modes of the $\mathrm{PbI}_{3}{ }^{-}$inorganic sublattice $\left(\nu_{\mathrm{TO}}\right)$.

We compare the dielectric function of $\mathrm{MAPbI}_{3}$ with those of a polar liquid $\left(\mathrm{H}_{2} \mathrm{O}\right.$, Fig. 1d), a common ferroelectric solid $\left(\mathrm{SrBi}_{2} \mathrm{Ta}_{2} \mathrm{O}_{9}\right.$, Fig. 1e) and a polar semiconductor (GaAs, Fig. 1f). A comparison between Fig. 1c and Fig. 1d reveals similarity between reorientational relaxation of $\mathrm{H}_{2} \mathrm{O}$ and $\mathrm{CH}_{3} \mathrm{NH}_{3}{ }^{+}$in the gigahertz region, yet this range is less relevant for the understanding of large polaron behaviour, whose properties depend on the terahertz response of the $\mathrm{PbI}_{3}^{-}$sublattice in $\mathrm{MAPbI}_{3}$ (refs. ${ }^{2,3}$ ). Note that the dielectric function reflects the material transverse response with zero momentum vector $(\mathbf{k}=0)$, whereas polaron formation results from charge coupling to higher-frequency longitudinal optical (LO) phonon modes at higher momentum $(\mathbf{k}>0)^{2,3}$. The LO phonon-absorption spectrum is given by $\operatorname{Im}[-1 / \varepsilon(\nu)]$, shown in green (magnified by $\times 20$ relative to the other plots) in Fig. 1c, with a main peak at $\nu_{\mathrm{LO}}=4.0 \mathrm{THz}$.

Moving to the terahertz region, we observe that for GaAs (Fig. 1f) there is a small increase in $\varepsilon_{1}$ by $18 \%$ across the narrow TO resonance, $\Delta \nu_{\mathrm{TO}} / \nu_{\mathrm{TO}}=0.009\left(\Delta \nu_{\mathrm{TO}}\right.$, full-width at half-maximum of the TO resonance). In contrast, $\mathrm{MAPbI}_{3}$ features a large increase in $\varepsilon_{1}$ by one order of magnitude and the TO resonances, peaking at $\nu_{\mathrm{TO}}=1.85 \mathrm{THz}$ and $0.96 \mathrm{THz}$, are much broader $\left(\Delta \nu_{\mathrm{TO}} / \nu_{\mathrm{TO}}=0.2-0.3\right)$. This response is similar to that of $\mathrm{SrBi}_{2} \mathrm{Ta}_{2} \mathrm{O}_{9}$ in which $\varepsilon_{1}$ increases by $1-2$ orders with $\Delta \nu_{\mathrm{TO}} / \nu_{\mathrm{TO}}=0.4$. In the following, we focus on this similarity and discuss how the ferroelectric-like response can lead to the formation of a ferroelectric large polaron. The dielectric functions of other LHPs are very similar in the terahertz region, independent of cation type ${ }^{2,6}$, and the following discussion applies to LHPs in general.

A ferroelectric material possesses macroscopic electrical polarization due to ordering of microscopic dipoles in a crystal lacking centre symmetry. Common to most ferroelectrics is the involvement of a TO phonon that softens as the system approaches the ferroelectric transition ${ }^{7}$. Here 'softening' and the corresponding 'soft mode' refer to the decrease in frequency (towards zero) of $\nu_{\mathrm{TO}}$; this results in the disappearance of restoring force in a vibrational coordinate and thus leads to structural instability and phase transition. In 'relaxor' ferroelectrics with crystalline disorder and polar nanodomains ${ }^{8}$, softening can be responsible for the transition from a non-polar phase to a polar one, in which the randomly distributed polar nanodomains can order under the action of an external electric field ${ }^{8}$. One of the key consequences of the soft mode is the large increase in $\varepsilon_{1}$ as the frequency decreases over the broad TO resonance ${ }^{7,8}$ as observed in $\mathrm{MAPbI}_{3}$ (Fig. 1c).

Another indication of soft-mode behaviour in LHPs is the quasi-elastic central peak near zero frequency ${ }^{7,8}$ seen in the low-frequency Raman spectra of LHPs $^{4}$. Moreover, giant photostriction has been measured in LHPs $^{9}$ - a phenomenon related to the large electrostriction ${ }^{8}$ observed in ferroelectrics, which is due to the large dipole moments of polar nanodomains and their dependence on electric field. Ferroelectric behaviour has also recently been confirmed in $\mathrm{MAPbI}_{3}$ in the tetragonal phase $\mathrm{e}^{10,11}$. Although macroscopic ferroelectricity may be absent, dynamic symmetry breaking and local alignment of polarization can still exist in $\mathrm{LHPs}^{12,13}$. This dynamical disorder alters the potentialenergy surface for atomic displacements in a unit cell, which shows multiple minima rather than a single minimum close to the equilibrium position. As a result, under sufficiently intense electric field, $P$ can flip between different minima, changing in sign and increasing by one or more orders of magnitude in absolute value (Fig. 1b). The $P-E_{\mathrm{f}}$ response can also show hysteresis (dashed lines in Fig. 1b) if the potential barrier between the two minima is above the thermal energy $k T$.

This result has an immediate impact on the formation mechanism of large polarons. In fact, in a conventional polar solid, the dipole moment (and $P$ ) induced by an injected charge carrier is linear with $E_{\mathrm{f}}$ and hence diminishes as distance from the injected charge increases ${ }^{2,3}$ (Fig. 1a, right). However, in a ferroelectric-like material, 
the local electric field (reaching values of $0.1-1 \mathrm{MV} \mathrm{cm}^{-1}$ within about $20 \mathrm{~nm}$ from the charge) is intense enough ${ }^{11}$ to induce collective polarization and trigger local phase transitions to form and order polar nanodomains surrounding the charge (Fig. 1b, right). The result is a ferroelectric large polaron. Note that our proposal differs from a conventional ferroelectric phenomenon in two ways: (1) it is dynamic and transient, vanishing when the charge is removed; and (2) no unidirectional, macroscopic polarization occurs, but rather a local microscopic ordering with radial symmetry centred on the charge.

The formation and/or ordering of polar nanodomains surrounding a charge carrier in a ferroelectric large polaron introduces much larger screening of the Coulomb potential than a conventional large polaron does. In addition, the collective polarization of the nanodomains may result in a potential barrier towards charge recombination. A more quantitative understanding of the ferroelectric large polaron is challenging because collective polarization coupled with soft phonons demands theoretical treatments beyond the harmonic approximation. Although the dielectric function is informative, it is limited to transverse responses at zero momentum $(\mathbf{k}=0)$, but response of phonons to a charge carrier is a local process described as superposition of dielectric responses at $\mathbf{k} \neq 0$. Experiments to determine dielectric function at $\mathbf{k} \neq 0$ based on, for instance, electron scattering, are needed. The analysis presented here also suggests a new design principle for defect-tolerant semiconductors based on soft, hybrid and nanostructured materials with local and/or dynamic symmetry breaking and ferroelectric-like dielectric responses.

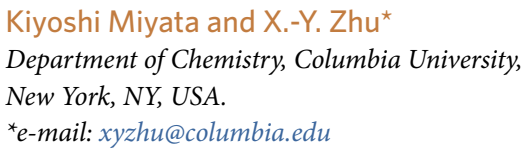

Published online: 23 April 2018

https://doi.org/10.1038/s41563-018-0068-7

References

1. Zhu, X. \& Podzorov, V. J. Phys. Chem. Lett. 6, 4758-4761 (2015).

2. Miyata, K. et al. Sci. Adv. 3, el701217 (2017).

3. Sendner, M. et al. Mater. Horizons 3, 1-8 (2016).

4. Yaffe, O. et al. Phys. Rev. Lett. 118, 136001 (2017).

5. Anusca, I. et al. Adv. Energy Mater. 7, 1700600 (2017).

6. Miyata, K., Atallah, T. L. \& Zhu, X.-Y. Sci. Adv. 3, e1701469 (2017).

7. Buixaderas, E., Kamba, S. \& Petzelt, J. Ferroelectrics 308, 131-192 (2004)

8. Cowley, R. A., Gvasaliya, S. N., Lushnikov, S. G., Roessli, B. \& Rotaru, G. M. Adv. Phys. 60, 229-327 (2011).

9. Zhou, Y. et al. Nat. Commun. 7, 11193 (2016).

10. Strelcov, E. et al. Sci. Adv. 3, el602165 (2017).

1. Rakita, Y. et al. Proc. Natl Acad. Sci. USA 114, E5504-E5512 (2017).

12. Beecher, A. N. et al. ACS Energy Lett. 1, 880-887 (2016).

13. Etienne, T., Mosconi, E. \& De Angelis, F. J. Phys. Chem. Lett. 7, 1638-1645 (2016).

14. Segelstein, D. J. The Complex Refractive Index of Water. M.Sc. thesis, Univ. of Missouri-Kansas City (1981).

15. Kamba, S. et al. Appl. Phys. Lett. 81, 1056-1058 (2002).

16. Palik, E. D. Handbook of Optical Constants of Solids (Academic, Orlando, FL, 1985).

\section{Acknowledgements}

X.Y.Z. acknowledges support by the Vannevar Bush Faculty Fellowship through Office of Naval Research grant no. N00014-18-1-2080 during the writing of this paper. K.M. acknowledges the Japan Society for the Promotion of Science for financial support.

\title{
The influence of the Rashba effect
}

\author{
Heavy atoms and crystal or inversion symmetry breaking may promote Rashba effects in halide perovskites. Sam \\ Stranks and Paulina Plochocka propose experiments to assess the existence of these effects and their implications \\ on the photophysics of perovskites.
}

$\mathrm{n}$ semiconductors, electrons and holes are typically described in an effective mass approximation with spin-degenerate parabolic dispersion around extrema points of the valence and conduction bands. The presence of heavy atoms in the crystal leads to spin-orbit coupling, which, combined with breaking of the inversion or crystal symmetry, gives rise to Rashba-type effects ${ }^{1}$. As a consequence, the spin degeneracy in $k$-space is lifted and the valence band maxima and/or conduction band minima are shifted away from the symmetry points in the Brillouin zone.

It has been proposed that metal halide perovskites possess both necessary properties for Rashba-type effects ${ }^{2}$. These systems, such as the archetypical methylammonium lead iodide, exhibit very strong spin-orbit coupling due to the presence of heavy ions such as lead or iodide in the crystal structure. In contrast with many semiconductors, the spin-orbit coupling in these halide perovskites is stronger in the conduction band than in the valence band ${ }^{3}$. Researchers have proposed various means by which the symmetry is broken in the threedimensional centrosymmetric perovskite crystal structures, including octahedral tilting of the inorganic lead-halide cage and dynamic rotation of the organic cation on the timescale of a few picoseconds ${ }^{4}$. The latter breaks the symmetry on a very local scale but preserves global centrosymmetry, which is consistent with measurements of phonon dispersions that reveal fluctuating symmetry-broken domains on a scale of $\sim 1-3 \mathrm{~nm}$ in size $\mathrm{e}^{5}$.

Rashba-type effects lead to the intriguing possibility that the fundamental bandgap may be indirect in these halide perovskite systems $^{2,3}$ (Fig. 1a). The indirect band is only slightly shifted in $k$-space $\left(k_{0}\right)$ and is predicted to be at a slightly lower energy $(\Delta E \sim 50 \mathrm{meV})^{3}$ than the direct band. This could have an enormous impact on the dynamics of charge carriers: light absorption may proceed through direct transitions, but recombination of cooled carriers may be slowed by the band extrema shift in $k$-space, leading to a forbidden indirect transition ${ }^{2}$.

There are still only a few reports that provide experimental indications for these theoretical predictions. Time-resolved photoconductivity measurements have recently been used to show that excitation below the direct gap into an indirect band leads to the photogeneration of mobile carriers, which exhibit slower recombination than carriers generated by excitation into the direct band ${ }^{6}$. These results are supported by very recent observations of a circular photogalvanic effect: electric current is generated in a thin halide perovskite film excited with circularly polarized light below the optical bandgap. Moreover, left- and right-handed excitation induces photocurrents in opposite directions, thus providing direct evidence for phototransport in spin-split bands (Fig. 1c) ${ }^{7}$. In another experiment, hydrostatic pressure was applied to perovskite samples to decrease the Rashba effect and shift the system to a more direct bandgap, causing a rapid increase in the recombination rate and an activation energy for recombination of $\Delta E \sim 60 \mathrm{meV}$ (ref. ${ }^{8}$ ). Polarization- and angle-resolved photoemission spectroscopy has been used to extract the dispersion of the highestenergy valence band in a $\mathrm{MAPbBr}_{3}$ single crystal, revealing spin splitting ${ }^{9}$. We note that this photoemission technique is surfacesensitive and therefore may exaggerate Rashba-type effects by selectively probing 


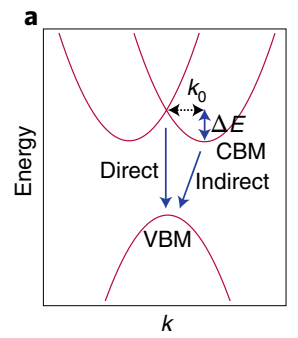

b

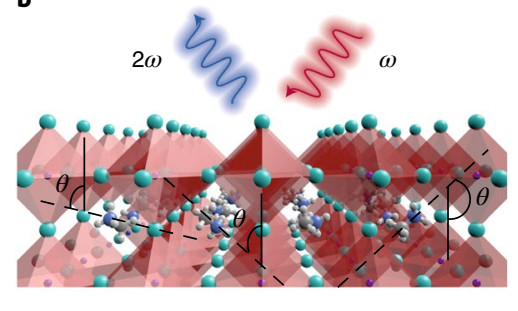

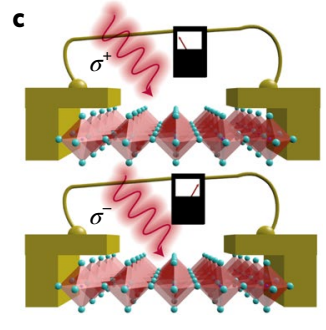

c

Fig. 1 | Rashba effects in halide perovskites and selected experiments to observe them. a, Schematic band diagram showing the bands slightly shifted in $k$-space by $k_{0}$. An indirect bandgap with an energy $\Delta E \sim 50 \mathrm{meV}$ below the direct gap is introduced ${ }^{3,6,8}$. VBM, valence band maximum; CBM, conduction band minimum. $\mathbf{b}$, Schematic of the second-harmonic generation effect in methylammonium lead iodide, where the crystal inversion symmetry is broken due to dynamic rotation of the cations. Red octahedra with light-blue spheres represent the inorganic lead-halide cage, and the blue and grey spheres represent the organic cations. The random orientation of the cations in each unit cell is marked by their different angles $\theta$. The incident light of frequency $\omega$ (red wavy arrow) interacts with the sample and generates light of frequency $2 \omega$ (blue wavy arrow). c, Schematic of circular photogalvanic measurements with polarized incident light (wavy arrows, with either $\sigma^{+}$or $\sigma^{-}$polarization). Electrodes are shown in yellow.

surface sites at which symmetry is more likely to be broken than the bulk.

The few experimental reports published to date have not yet settled the debate over the presence of the Rashba effect; hence, we now consider which experiments are potential 'smoking guns' to validate or falsify theoretical predictions of the Rashba-type splitting in halide perovskites. In bulk crystals, second-harmonic generation (SHG) with an electric dipole symmetry response is a nonlinear optical process in which a photon with frequency $\omega$ is upconverted to a photon with doubled frequency $(2 \omega)$ only in materials with broken inversion symmetry (Fig. 1b). This optical technique can therefore be used to probe the crystal symmetry. Furthermore, it could be performed with micrometre spatial resolution to determine whether local grain-to-grain heterogeneity, surfaces or grain boundaries lead to local inversion symmetry breaking. Another elegant way to probe Rashba-type couplings is to exploit the simple spin-charge conversion and the introduction of an unbalanced spin population (for instance, through circularly polarized light), thereby motivating further experiments along the lines of circular photogalvanic (Fig. 1c), spin galvanic effect and magneto-gyrotropic measurements ${ }^{10}$.

Rashba-type effects may also be accessed through measurements of the exciton fine structure. In semiconductors, the exchange interaction splits the dark and bright excitonic states, with the dark state being the lowest one. The bright states can be split further by lowering the symmetry of the crystal. It was recently proposed that the Rashba effect could affect the order of the states in colloidal $\mathrm{CsPbBr}_{3}$ nanocrystals - making a bright triplet the ground state - and thus strongly enhance the exchange splitting of the bright states ${ }^{11}$. It should be noted that quantum confinement and surface effects may significantly contribute to symmetry breaking in these nanocrystal systems. Hence, low-temperature measurements of the exciton fine structure in larger three-dimensional perovskites crystals will be required to isolate exchange spitting originating purely from the Rashba effect.

Still, an important question remains: if there is indeed a Rashba effect, what role will it play in the operation of perovskite optoelectronic devices and solar cells? Charge-carrier recombination is influenced not only by the direct or indirect nature of the bandgap, but also by a number of other factors, including carrier trapping ${ }^{12}$ and possible polaronic screening effects ${ }^{13}$. The relative contributions still need to be decoupled, but it is likely that traps are the main factor affecting recombination in most perovskite solar cells to date ${ }^{12}$. As the material quality further improves and the number of traps is reduced, the Rashba effect may play a more significant role: the indirect bandgap may even cause an unwanted $\sim 50 \mathrm{mV}$ voltage loss in device performance. Nevertheless, it is likely that the net symmetry breaking in highly crystalline bulk films - those used in planar heterojunction solar cells - will be negligible, thus reducing the contributions of the Rashba effect on recombination. Indeed, in typical three-dimensional solar cell materials, recombination rates seem to be primarily direct and radiative, at least at high charge densities ${ }^{14}$.

Another important question is whether we can exploit this effect in novel device applications. The Rashba parameter $\alpha=2 \Delta E / k_{0}$ in halide perovskites - estimated from angle-resolved photoemission spectroscopy measurements and predicted theoretically — is of the order a few eV $\AA$, which is comparable with the highest values reported for bulk crystals, thus rendering them promising for spintronics ${ }^{10}$. It has also been proposed ${ }^{15}$ that the strength of Rashba splitting can be controlled via an external electric field in the direction perpendicular to the plane. Such an approach may find application in spin field-effect transistors to generate, control and detect spin current and prepare spin states. As demonstrated in a recent study on colloidal perovskite nanocrystals ${ }^{11}$, Rashba effects may be more prominent if three-dimensional centrosymmetric structures are replaced by nanocrystals or by two- or quasi-twodimensional analogues in solar cells or light-emitting devices ${ }^{16}$. Thus, tactical materials engineering may allow controlled exploitation of Rashba physics to tailor the behaviour of perovskite-based devices.

Studies of Rashba-type effects in perovskites are still in their early stages, and further understanding of the phenomena and impact on charge-carrier recombination and transport will allow us to fully remove or maximally exploit the effect, as desired.

Samuel D. Stranks ${ }^{1 *}$ and Paulina Plochocka ${ }^{2 *}$ ${ }^{1}$ Cavendish Laboratory, University of Cambridge, Cambridge, UK. ${ }^{2}$ Laboratoire National des Champs Magnétiques Intenses, CNRS, Toulouse, France.

*e-mail:sds65@cam.ac.uk;

paulina.plochocka@lncmi.cnrs.fr

Published online: 23 April 2018

https://doi.org/10.1038/s41563-018-0067-8

References

1. Rashba, E. Sov. Phys. Solid State 2, 1224-1238 (1960).

2. Zheng., F., Tan, L. Z., Liu, S. \& Rappe, A. M. Nano Lett. 15, 7794-7800 (2015)

3. Azarhoosh, P., McKechnie, S., Frost, J. M., Walsh, A. \& van Schilfgaarde, M. APL Mater. 4, 091501 (2016).

4. Etienne, T., Mosconi, E. \& De Angelis, F. J. Phys. Chem. Lett. 7, 1638-1645 (2016)

5. Beecher, A. N. et al. ACS Energy Lett. 1, 880-887 (2016).

6. Hutter, E. M. et al. Nat. Mater. 16, 115-120 (2017).

7. Niesner, D. et al. Preprint at https://arxiv.org/abs/1703.08740 (2017).

8. Wang, T. et al. Energy Environ. Sci. 10, 509-515 (2017).

9. Niesner, D. et al. Phys. Rev. Lett. 117, 126401 (2016).

10. Kepenekian, M. \& Even, J. J. Phys. Chem. Lett. 8, 3362-3370 (2017).

11. Becker, M. A. et al. Nature 553, 189-193 (2018).

12. Stranks, S. D. ACS Energy Lett. 2, 1515-1525 (2017).

13. Zhu, H. et al. Science 353, 1409-1413 (2016).

14. Davies, C. L. et al. Nat. Commun. 9,293 (2018)

15. Kim, M., Im, J., Freeman, A. J., Ihm, J. \& Jin, H. Proc. Natl Acad. Sci. USA 111, 6900-6904 (2014).

16. Zhai, Y. et al. Sci. Adv. 3, e1700704 (2017). 


\section{Clues from defect photochemistry}

Charge carriers in metal halide perovskites seem to be only marginally affected by defect-related trap states. Filippo De Angelis and Annamaria Petrozza suggest that the key to this behaviour lies in the redox chemistry of halide defects.

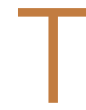
he efficiency of a solar cell is intimately related to the fate of photogenerated electron-hole pairs in the absorber layer. The interaction and capture of charge carriers through defects acting as recombination centres may significantly limit the solar cell's open-circuit voltage and thus depress its overall performance.

The efficient charge generation and collection in devices based on lead-halide perovskites are, to a first approximation, related to the extremely long lifetimes of photogenerated carriers. These lifetimes depend on the intrinsic nature of the charge carriers - particularly the electronic band structure and charge-lattice interactions and on the optoelectronic quality of the semiconductor (that is, the amount of traps/defects interacting with the carriers). Careful control of crystal growth is required to obtain semiconductors with high purity (typical trap densities of $10^{8} \mathrm{~cm}^{-3}$ for crystalline $\mathrm{Si}$ ) and, therefore, high efficiency. In contrast, polycrystalline thin films of metal halide perovskites made from solution at low temperature show excellent performance even at high trap densities $\left(\sim 10^{16} \mathrm{~cm}^{-3}\right.$ for methylammonium lead tri-iodide $\mathrm{MAPbI}_{3} ;$ ref. $\left.^{1}\right)$. Trap energy levels spanning $\sim 0.3 \mathrm{eV}$ above and below the band edges have also been found in $\mathrm{MAPbI}_{3}$ single crystals ${ }^{2}$. The pressing questions are: what is the nature of these defects, and what are the photochemical processes that govern them?

Among native point defects in bulk $\mathrm{MAPbI}_{3}$ (ref. ${ }^{3}$ ), those related to $\mathrm{Pb}$ and MA show the lowest formation energy, pinning the Fermi level approximately at mid-gap $(0.72 \mathrm{eV}$ over a $1.58 \mathrm{eV}$ calculated bandgap) ${ }^{4}$. Such defects are shallow traps and may only be moderately active $^{4}$; although their presence might be thermodynamically favoured, they are less likely to form in the bulk crystal owing to their high migration activation energies. Defects related to iodine atoms placed in usually unoccupied lattice sites, called interstitial iodine defects, instead introduce deep levels in the $\mathrm{MAPbI}_{3}$ bandgap $^{5}$ and are expectedly active by virtue of their low migration energies ${ }^{6}$.

We believe that the key to explaining the so-called defect tolerance in this class of semiconductors lays in the peculiar a

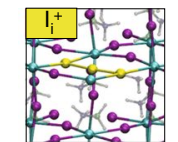

b
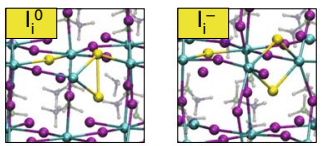

d

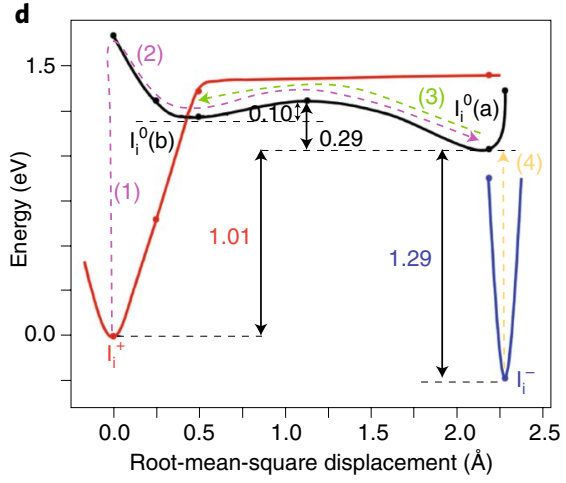

Photon energy (eV)

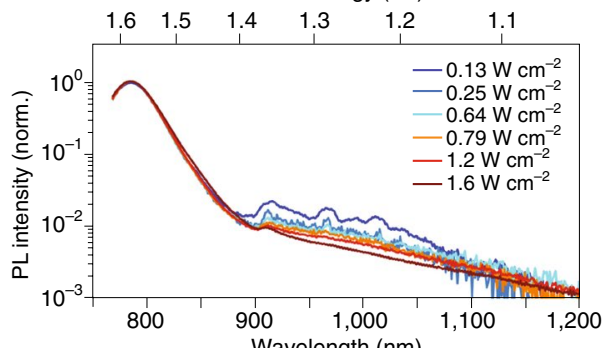

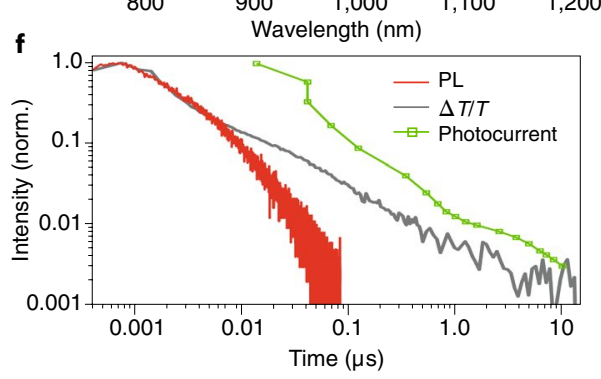

Fig. 1 | Interstitial iodine defects as hole and electron traps. a-c, Local geometrical structures of interstitial iodine in its positive (a), neutral (b) and negative (c) charge states. Relevant iodine atoms involved in the trapping/detrapping processes are highlighted in yellow. Other iodine atoms, lead atoms and methylammonium molecules are shown in magenta, cyan and grey, respectively. $\mathbf{d}$, Configuration diagrams (energy versus structural coordinates) of interstitial iodine in its positive (solid red), neutral (solid black) and negative (solid blue) charge states, versus the root-mean-square displacement of the highlighted iodine atoms with respect to their position in $\mathrm{I}_{i}^{+}$. The positive (negative) state energy is referenced to the valence band maximum (conduction band minimum). Also shown are possible pathways for thermally activated electron trapping (dashed magenta lines, 1,2 ); thermally activated electron detrapping (dashed green line, 3); and radiative hole trapping/detrapping (dashed orange line, 4). e,f, Spectroscopic signatures of trap dynamics. e, Steady-state photoluminescence (PL) spectra of $\mathrm{MAPbl}_{3}$ recorded at increasing excitation fluence. $\mathbf{f}$, Photoluminescence dynamics probed at $1.56 \mathrm{eV}$, compared with the photobleach dynamics probed at $1.61 \mathrm{eV}$ (optical gap of the semiconductor) and the photocurrent dynamics measured on a perovskite layer with two lateral Ohmic contacts. Photocurrent data are from ref. ${ }^{9}$.Credit: adapted from ref. ${ }^{4}$, Royal Society of Chemistry

photochemical properties of such interstitial defects. The charge trapping-detrapping processes in $\mathrm{MAPbI}_{3}$ can be understood by looking at the configuration diagram shown in Fig. 1, where we report the energy of interstitial iodine $\left(\mathrm{I}_{\mathrm{i}}\right)$ within a tetragonal $\mathrm{MAPbI}_{3}$ supercell against a collective structural parameter ${ }^{4} . \mathrm{I}_{\mathrm{i}}$ can have various charge states: a positive $\mathrm{I}_{i}^{+}$ state, showing the trimer structure of a coordinated $\mathrm{I}_{3}^{-}$molecule (yellow spheres in Fig. 1a), which acts as an electron trap with a $(+/ 0)$ transition $1.01 \mathrm{eV}$ above the valence band; a neutral $\mathrm{I}_{\mathrm{i}}^{\circ}$ state (Fig. $1 \mathrm{~b}$ ), where the defect iodine atoms form a bridged dimer geometry; and a negatively charged $\mathrm{I}_{\mathrm{i}}^{-}$state (Fig. 1c), which behaves as a hole trap with a (0/-) transition $1.29 \mathrm{eV}$ below the conduction band (or $0.29 \mathrm{eV}$ above the valence band), showing a similar structure to that of $I_{i}^{\circ}$. As shown in Fig. 1d, the energy minima of these states have significantly different structures. This means that when the defect traps or releases a charge, significant structural rearrangement may occur. Trapping of an electron at $\mathrm{I}_{\mathrm{i}}^{+}$(' 1 ' in Fig. 1d) induces a strong structural relaxation that drives the system to the $\mathrm{I}_{\mathrm{i}}^{\circ}$ (a) global minimum of the neutral charge state (' 2 ' in Fig. 1d). This process is thermodynamically favoured and features a small energy barrier of $0.1 \mathrm{eV}$ above the local $\mathrm{I}_{\mathrm{i}}^{\circ}(\mathrm{b})$ minimum. To release the trapped electron and reform $\mathrm{I}_{\mathrm{i}}^{+}$(' 3 ' in Fig. 1d), the 
system must overcome a $0.29 \mathrm{eV}$ barrier on the neutral curve. Electron detrapping will thus be a thermally activated process. Hole trapping causes a transition from $\mathrm{I}_{\mathrm{i}}^{-}$to $\mathrm{I}_{\mathrm{i}}{ }^{\circ}$ ('4' in Fig. 1d), thus proceeding with a small geometrical rearrangement and no energy barriers. As such, hole trappingdetrapping is expected to be relatively fast. This analysis suggests that electron release by interstitial iodine will require additional thermal energy, and it is thus expected to be a slow process that may induce energy losses through non-radiative deactivation. In contrast, holes trapped at $\mathrm{I}_{\mathrm{i}}^{0}$ can be easily released and contribute to relatively rapid radiative recombination. Since interstitial iodine traps are sub-bandgap states, this means that radiative recombination - light emission - should be observed at energies below the bandgap (1.29 against a $1.58 \mathrm{eV}$ calculated bandgap, Fig. 1d).

Steady-state room-temperature photoluminescence spectra of a typical $\mathrm{MAPbI}_{3}$ thin film, in fact, reveal emission in the 1.35-1.15 eV range, below the band-toband radiative emission at $\sim 1.6 \mathrm{eV}$ (Fig. 1e). Such emissive states have been found to be populated and depopulated in a time window shorter than $1 \mathrm{~ns}\left(\mathrm{refs}^{7,8}\right)$, which is in line with the behaviour expected from the fast radiative recombination of trapped holes described above. At high excitation energy the amount of photogenerated holes saturates these traps, which have no time to recombine radiatively; as a result, subbandgap emission is quenched.

By performing transient-absorption experiments in the nanosecondmicrosecond time window (Fig. 1f), we have observed the dynamics relating to band edge photocarriers, which show a fast charge recombination process matching the dynamics of band-to-band radiative recombination. Yet we have also observed a second component with a very long decay of tens of microseconds. This does not correspond to any radiative feature, although it is traceable by looking at photocurrent dynamics ${ }^{9}$. We assign such slow components to the non-radiative recombination of electrons trapped by $\mathrm{I}_{i}^{+}$with free holes (' 3 ' in Fig. 1d).

Owing to their long-living nature, electron traps, once filled, will remain inactive for a long time (up to several microseconds), thus becoming essentially not harmful to charge recombination. However, the rapid recombination of trapped holes demonstrated by photoluminescence experiments reveals the higher activity of these sub-bandgap energy levels, which may cause voltage losses ${ }^{10}$. Importantly, it has been shown that hole traps can be converted to kinetically inactive electron traps by exposing the samples to mild oxidizing conditions ${ }^{11}$ or $\mathrm{O}_{2}$ (ref. ${ }^{12}$ ). As a result, most of these converted traps will have little impact on the charge recombination photophysics of lead-halide perovskites. The mechanisms described here, which explain the apparent defect tolerance of lead-halide perovskites, thus depend on the peculiar redox chemistry of iodine defects related to retarded charge recombination in dyesensitized solar cells based on the iodide/ tri-iodide redox shuttle ${ }^{13}$.

Based on this analysis, one may be tempted to recommend a perovskite growth under iodine-poor conditions, to minimize the presence of interstitial iodine defects. However, tuning the chemical potential of a species in solution by a factor of $\sim 0.06 \mathrm{eV}$ implies a variation in its activity (concentration) of one order of magnitude; an impracticably large non-stoichiometric precursor formulation is therefore needed to cause a significant reduction in interstitial iodine defects. Instead, it has been shown that working in a slightly iodine-rich environment (for instance $10 \%$ excess MAI) can benefit crystal growth ${ }^{14}$, while essentially not perturbing the density of iodinerelated defects. Mastering the conversion of interstitial iodine defects from hole traps to electron traps is therefore a more promising way to optimize the behaviour of such materials. Trap passivation may be further promoted in mixed-halide perovskites by saturation of interstitial iodine sites by smaller halides, such as $\mathrm{Cl}$ and $\mathrm{Br}$, which transform these mixed-halide interstitials into shallow traps ${ }^{4}$. Variations in halide composition may thus perturb such a system of defects, and this is likely to be the key to success for mixed-halide perovskites ${ }^{15}$.

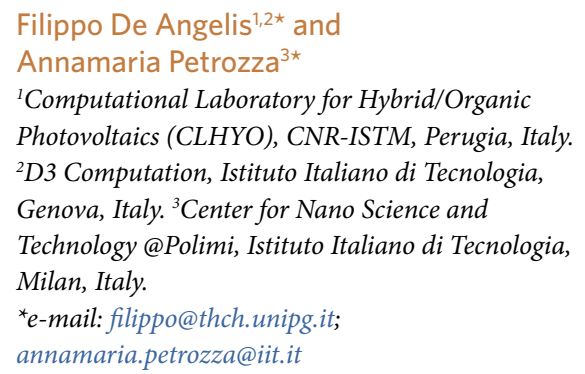

Published online: 23 April 2018

https://doi.org/10.1038/s41563-018-0069-6

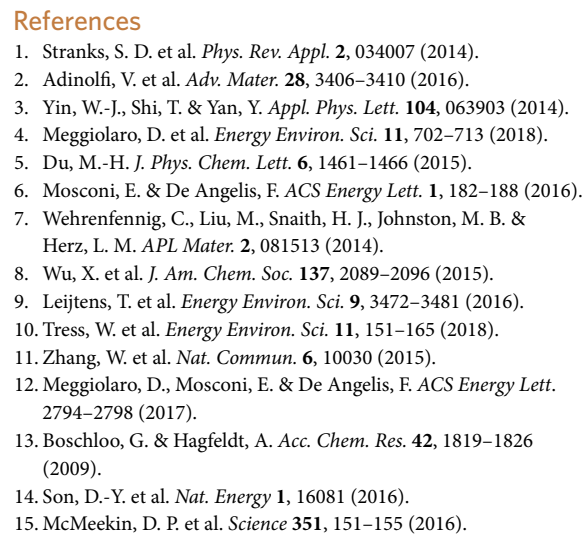

